117 | janvier - mars 2002

Les religions afro-américaines : genèse et développement dans la modernité

\title{
Le «Cercle Dieu et vérité » un mouvement panthéiste entre les populations d'origine africaine de Recife (1929/1968)
}

\section{Marion Aubrée}

\section{OpenEdition}

\section{Journals}

Édition électronique

URL : http://journals.openedition.org/assr/2482

DOI : 10.4000/assr.2482

ISSN : $1777-5825$

Éditeur

Éditions de l'EHESS

Édition imprimée

Date de publication : 1 janvier 2002

Pagination : 81-99

ISBN : 2-222-96715-5

ISSN : 0335-5985

\section{Référence électronique}

Marion Aubrée, «Le « Cercle Dieu et vérité » un mouvement panthéiste entre les populations d'origine africaine de Recife (1929/1968) », Archives de sciences sociales des religions [En ligne], 117 I janvier mars 2002, mis en ligne le 18 novembre 2005, consulté le 30 avril 2019. URL : http://

journals.openedition.org/assr/2482 ; DOI : 10.4000/assr.2482 


\section{LE « CERCLE DIEU ET VÉRITÉ » UN MOUVEMENT PANTHÉISTE ENTRE LES POPULATIONS D'ORIGINE AFRICAINE DE RECIFE $(1929 / 1968)$}

Nous présentons ici, à partir de l'analyse d'un mouvement socioreligieux urbain auto-désigné comme "panthéiste» qui s'est développé dans la ville de Recife sur une quarantaine d'années, les premiers développements d'une étude dont l'objectif est de comprendre la spécificité de l'homo religiosus brésilien actuel, de voir comment celle-ci s'est construite et quelles peuvent être les répercussions de certaines structures antérieures sur des attitudes et des comportements présents qui ressortissent à ce que de nombreux auteurs appellent aujourd'hui les "Nouveaux Mouvements Religieux » ${ }^{1}$.

Le Brésil est un pays où l'histoire a essaimé des courants de pensée et des formes de croyance issus d'horizons culturels diversifiés. Les mythes et les symboles ainsi véhiculés se sont le plus souvent structurés en des systèmes autonomes d'expression religieuse populaire qui, compte tenu des transformations du contexte historique, n'ont cessé de cohabiter et, parfois, de se fondre, donnant ainsi naissance à des mouvements religieux ou mystiques nouveaux ou revitalisant des formes confessionnelles ou ésotériques plus anciennes.

Sur le plan socioreligieux, on a observé au long des deux derniers siècles la permanence au Brésil de quelques structures symboliques fortes qui resurgissent régulièrement puis s'estompent pour réapparaître quelques décennies plus tard, à la faveur d'un contexte social, voire politique, plus favorable. On remarque, en particulier, malgré l'hégémonie historique du catholicisme et l'introduction plus tardive de quelques autres doctrines d'origine européenne dans lesquelles le spirituel est

\footnotetext{
${ }^{1}$ Pour ma part, je les appellerai ici « Nouvelles Spiritualités » dans la mesure où la dimension individuelle qui se profile dans la «quête» du croyant potentiel induit une dynamique de liaison, parfois exclusive, entre une divinité ou une «force » supérieure et l'individu en état de recherche. Cette dynamique individualisante semble, au moins au Brésil, être un phénomène relativement nouveau dans une société où le propre de la quête mystique était jusqu'à récemment de se structurer autour d'une figure charismatique et d'une dimension communautaire.
} 
mis en valeur à l'exclusion de tout symbole lié à la matière ${ }^{2}$, une nette tendance à donner une valeur symbolique forte et à sacraliser des éléments naturels et matériels tels que l'eau, la terre et ses productions (plantes de toutes sortes), les pierres et les astres. La réémergence régulière de ce substrat culturel témoigne du fait que la société brésilienne demeure profondément imprégnée par la vision du monde portée par les cultures issues des apports amérindien et africain. Or, ces cultures, qui ne sont pas minoritaires et qui ont, tout autant que l'apport européen, fondé la spécificité culturelle brésilienne, n'ont jamais bénéficié d'une reconnaissance historique de la part des élites.

Les mouvements socioreligieux ruraux, qualifiés de «messianiques » ou de « millénaristes ", qui ont eu le plus souvent pour cadre l'intérieur aride (sertão) du Nordeste brésilien, ont fait l'objet de nombreuses études ${ }^{3}$. Dans cette même région, le milieu urbain a produit lui aussi un brassage original entre des formes du croire plus populaires et plus marquées de matérialité et des façons de penser plus abstraites, liées à la vision de ceux qui dominaient le champ politique et intellectuel. Ces mouvements répondaient, pour certains d'entre eux, à une volonté de réunir en une vision du monde unifiée des éléments symboliques et rituels utilisés par des groupes appartenant à des couches sociales différenciées. Ils allaient ainsi dans le sens de l'affirmation de l'intégration nationale, une intégration chère aux élites ${ }^{4}$ mais également à un peuple tout aussi désireux d'articuler ses différences dans le grand creuset de la Nation.

Les tentatives faites du côté des couches plus aisées et lettrées pour réunir des éléments culturels qui pouvaient apparaître au premier abord comme antagonistes sont bien documentées. Ainsi, le spiritisme kardéciste et les cultes afro-brésiliens, même si leurs deux doctrines sont fondées sur un rapport étroit et une communication, rituellement élaborée, entre un monde visible et un monde invisible, envisagent la relation entre ces deux plans de façon tout à fait différente. Dans le premier, le support de l'attraction de l'invisible vers le visible est essentiellement spirituel ; au quotidien, il s'exprime par le biais de la médiumnité, par l'étude, par une conduite exemplaire, tandis que le rituel se déroule sous le signe du silence et du recueillement. Dans les cultes afro-brésiliens, au contraire, on atteint le plan invisible et on agit sur lui à travers la manipulation d'éléments matériels (pierres, plantes, animaux, corps humains, objets rituels) qui sont autant de messages pour les entités symboliques que l'on veut invoquer. Dans l'évolution kardéciste on n'a que faire de la Nature, seul compte l'Esprit, tandis que l'essence des orixás (divinités afro-brésiliennes) est intrinsèquement liée aux éléments naturels. Pourtant, l'umbanda est issue de ces deux univers de croyance. Son apparition correspond au besoin spirituel et à la volonté politique de quelques-uns de créer une troisième voie mieux susceptible, à leurs yeux, de servir l'élaboration d'une identité nationale en devenir. Ce cas a été bien documenté et analysé, entre autres par D. Brown (1986) et R. Ortiz (1978) dans leurs travaux sur le surgissement à Rio et l'expan-

\footnotetext{
2 Je pense en particulier à la doctrine spirite kardéciste qui a connu un succès sans précédent au Brésil depuis son introduction dans ce pays au milieu des années 1860 ; pour plus de détails sur ce sujet voir Marion Aubrée, François Laplantine, 1990.

${ }^{3}$ Voir, entre autres, Maria-Isaura Pereira de QueIroz,1968 ; Douglas TeIXeIra Monteiro, 1974 et Ralph Della Cava, 1970.

${ }^{4}$ Voir en particulier Alberto TORRES, 1933.
} 
sion à São Paulo de cette religion dont les codificateurs voulaient faire la « religion nationale ».

On connaît moins bien les mouvements issus de cercles d'origine plus modeste qui ont tenté de créer des " ponts » entre une vision sociale positiviste d'ordre et de progrès, véhiculée par les élites, et leur propre conception du monde qui faisait une place essentielle à l'intégration de l'homme au milieu naturel et au cosmos. Il faut aussi signaler que ceux-ci ont généralement duré moins longtemps que les premiers dans la mesure où ils étaient, comme tous les mouvements socioreligieux brésiliens issus des milieux populaires, très tributaires du charisme de leur fondateur, donc plus susceptibles de disparaître en même temps que celui-ci.

J'ai choisi d'aborder ce problème de la créativité religieuse des couches défavorisées et de leur capacité à articuler des éléments mystiques divers pour en faire une doctrine répondant à leurs aspirations tant spirituelles que socioculturelles, à partir d'un mouvement particulier qui appartient à ce second type. Il s'agit du « Cercle Dieu et Vérité », dont on sait, grâce aux nombreuses photos qui l'ont illustré, que ses fidèles étaient dans leur immense majorité noirs ou métis. Fondé dans la ville de Recife en 1929, il a fait l'objet sur une trentaine d'années de quelques publications rédigées soit par des psychiatres, membres de la Ligue d'Hygiène Mentale de Pernambuco et qui, à ce titre, visitaient tous les lieux de culte des quartiers pauvres, soit par des ethnographes, folkloristes et sociologues qui s’intéressaient aux attitudes et aux comportements particuliers des gens de leur région ou, plus largement, de leur pays.

Ce groupe n'existant plus à l'heure actuelle, il n'a pas été possible de trouver des informateurs, ex-fidèles panthéistes, pour recueillir une "mémoire vive ". C'est donc à partir de quatre auteurs que j'ai essayé de reconstituer le système symbolique de ce groupe tout à fait spécifique dont les caractéristiques doctrinales et les pratiques liturgiques s'éloignaient sensiblement des autres expressions religieuses du milieu environnant mais qui recèlent des éléments structurels que l'on retrouve aujourd'hui dans certaines expressions mystiques très contemporaines.

Les sources actuellement en ma possession sont les suivantes : d'abord, un article publié en 1933 par Pedro Cavalcanti, jeune psychiatre lié à la Ligue d'Hygiène mentale de Pernambuco fondée par le Dr. Ulysses Pernambucano. Cette même année Gilberto Freyre, dans son livre Casa Grande e Senzala ${ }^{5}$ fait une très brève mention de quelques caractéristiques de ce groupe dans une note de bas de page. En juin 1937, Vicente Lima publie le livre Xangô dont le dernier chapitre est consacré au Círculo Deus e Verdade. Enfin, Waldemar Valente, psychiatre et anthropologue, publie en $1966^{6}$ un long article dans lequel il reprend les descriptions et arguments de ses prédécesseurs pour les confronter à son propre travail de recherche au sein de cette communauté.

5 Traduction française de Roger BAstide: Maîtres et esclaves, Paris, Gallimard, 1952 et 1974.

6 «Panteismo em Pernambuco », dans 1'Annuaire de la Faculté de philosophie, 1962-1964, nº 7 , pp. $129-179$. 


\section{Diversité philosophique et religieuse à Recife}

Avant d'analyser ce cas spécifique il importe de présenter la dynamique qui a engendré la création de ce mouvement ainsi que le contexte socioreligieux dans lequel il a pris naissance. J'ai choisi la ville de Recife pour repérer des "ponts » entre les divers systèmes symboliques parce que j'en connais bien le contexte religieux et y ai réalisé de nombreuses enquêtes de terrain. Par ailleurs, le choix de cette ville se justifie par le fait qu'elle fut considérée pendant longtemps comme la capitale de la région Nordeste et a été le cadre de divers mouvements intellectuels et politiques qui ont laissé des traces dans l'Histoire du pays. Aujourd'hui on y trouve les institutions, mouvements religieux, sociétés secrètes et confréries les plus variés d'entre lesquels se détachent l'Église catholique, dans ses formes institutionnelle (catholicisme romain/théologie de la libération) et dévotionnelle (catholicisme populaire/Renouveau charismatique), les très nombreuses dénominations évangéliques ou néo-évangéliques, les sectes néo-pentecôtistes, les cultes afrobrésiliens et amérindiens de tous types, le spiritisme kardéciste, les syncrétismes bouddhistes, les francs-maçons, les Rose-Croix et un certain nombre de Nouvelles Spiritualités. Ces dernières, de même que les sectes néo-pentecôtistes et les syncrétismes bouddhistes, sont d'arrivée relativement récente mais tous les autres mouvements étaient déjà présents à Recife dans le premier quart du vingtième siècle. S'il semblait exister alors entre leurs différentes visions du monde des barrières infranchissables, le passage des uns aux autres qu'effectuaient certains individus créait sinon une compréhension mutuelle au moins des rapprochements inattendus.

\section{1 - Catholicisme et franc-maçonnerie}

Pour observer ces rapprochements, nous prendrons pour point de départ la période de bouleversements sociaux et politiques qui entoure la fin de l'Empire, l'avènement de la République et la Constitution de 1891, laquelle institue la séparation entre l'Église catholique et l'État. Elle nous offre en effet beaucoup d'éléments.

À cette époque, Recife était le siège de diverses institutions religieuses (le Séminaire d'Olinda) et laïques (la Faculté de Droit) qui participaient activement au bouillonnement intellectuel national. Le séminaire d'Olinda, fut fondé au

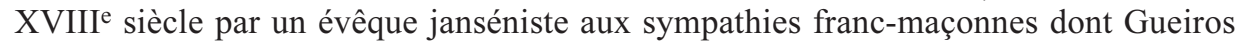
Vieira nous dit qu'il «transforma presque son séminaire en loge maçonnique » (1990, p. 47). De son côté, la Faculté de Droit donna au pays des abolitionnistes dont le plus célèbre fut Joaquim Nabuco qui, en 1880, prononça un discours sur la liberté de religion et refusa de voter le budget de soutien aux séminaires de l'Église catholique. On se trouvait alors face à deux courants se réclamant de la franc-maçonnerie, les uns plutôt catholiques et monarchistes et les autres libéraux, abolitionnistes et républicains. Cette importance de la franc-maçonnerie dans la ville a joué un rôle prépondérant dans la déflagration, en 1872, de la «Question religieuse » au cœur de laquelle se trouvait Dom Vital, évêque d'Olinda. Si j'insiste ici sur le rôle des francs-maçons c'est parce qu'on trouve dans leur doctrine des éléments qui méritent d'être soulignés et comparés aux composantes d'autres doc- 
trines également présentes dans le champ religieux brésilien. En effet, les Constitutions d'Anderson de 1723 (premier manifeste écrit d'un mouvement, la franc-maçonnerie spéculative, que Robert Gould (s.d.) fait remonter au XVe siècle) soulignent que les loges doivent enseigner à leurs membres l'œecuménisme religieux ainsi que la fraternité entre toutes les races, nationalités et classes sociales, par contre elles excluent péremptoirement les femmes de leurs confréries.

Tout au long de leur histoire les loges maçonniques ont été considérées comme des «sociétés secrètes» dont le Secret semblait bien gardé. À l'origine, la très grande majorité de leurs membres étaient des chrétiens, catholiques ou protestants selon la religion qui dominait dans leur pays d'appartenance. Mais en fonction des brassages d'idées qui se faisaient au sein des loges, une forme de pensée moins confessionnelle et plus philosophique s'imposa au fil des ans. Les francs-maçons adoptèrent une vision « panthéiste » du monde, c'est-à-dire une métaphysique de la Nature dans laquelle la divinité et le monde sont considérés comme émanant l'un de l'autre, ce qui permet d'envisager le monde dans sa diversité réelle et dans son unité ontologique. Si l'idée est ancienne ${ }^{7}$, le mot apparaît pour la première fois en 1706 dans les écrits d'un Irlandais, John Toland, appartenant lui-même à la mouvance maçonnique et dont l'ouvrage postérieur Pantheisticon (1720) contient les idées principales qui informeront les Constitutions d'Anderson. Il y parle de Dieu comme étant cette "énergie créatrice et ordonnatrice de toutes choses, tendant toujours à la fin la meilleure ». Il avait eu l'occasion de fréquenter les Écoles fondées à Leyde par Spinoza (1632-1677) où l'on enseignait la pensée de celui qui est aujourd'hui considéré comme le plus important jalon de cette tradition philosophique. Dès leur apparition en tant que corps organisés mais secrets, les francs-maçons ont inquiété les pouvoirs. Toutefois, le fait que de nombreux membres des classes dirigeantes aient adhéré à des Loges a permis un contrôle interne relativement efficace. L'Église catholique, de son côté, s'est rapidement inquiétée du contenu philosophique de cette pensée moniste qui récuse l'acte de création et le dualisme divin/humain qui en découle. Une première condamnation papale avait été signée par Clément XII en 1738, suivie par une autre bulle de Benoît XIV en 1751 qui, malgré son érudition et son ouverture, voyait dans l'œcuménisme prôné par les Loges un danger pour l'hégémonie de l'Église romaine. Au cours du XVIII ${ }^{\mathrm{e}}$ siècle la vision du monde panthéiste a été développée, en particulier en France, par les encyclopédistes dont il est avéré pour plusieurs d'entre eux qu'ils étaient francs-maçons. Par ailleurs, on fait généralement de Diderot l'un des représentants du panthéisme dit "naturaliste » ou "matérialiste » distinct de celui de Spinoza. Ces développements tendant à faire de Dieu une émanation de la Nature, ont alimenté les courants de la pensée libérale et individualiste qui ont fomenté la Révolution française. Ce qui devait encore renforcer les préventions de l'Église catholique contre la franc-maçonnerie et accentuer sa recherche de preuves dans sa tentative d'abattre ce qu'elle considérait comme une hérésie ${ }^{8}$. Les développements de l'ultramontanisme et le Concile Vatican I (1869) avaient clairement pour but de séparer définitivement la pensée catholique, apostolique et romaine, de la vision du monde considérée comme impure et déviante que constituait la franc-maçonnerie.

\footnotetext{
${ }^{7}$ On la fait remonter aux Stoïciens (4e /3e b.c) et même à Pythagore (6e b.c.).

8 Père Ferrer-Benimeli, Les archives secrètes du Vatican et la franc-maçonnerie, Paris, Dervy-Livres, 1989.
} 
Arrivées au Brésil, et à l'instar de ce qui se passait à Recife, toutes ces idées ont produit une fragmentation des élites. Au XIX $\mathrm{X}^{\mathrm{e}}$ siècle, les deux grandes Loges brésiliennes (Benedictinos et Labradio) recouvraient les deux versants philosophiques du panthéisme et, tant au Parlement qu'au Gouvernement, elles essayaient de s'approprier le maximum de pouvoir. La "Question Religieuse " est venue consommer la rupture. Les travaux d'Antonio Gouvea Mendonça (1984) ont clairement montré, par ailleurs, les liens qui se sont tissés entre francs-maçons et protestants tout au long du XIX ${ }^{\mathrm{e}}$ siècle; au point que Rui Barbosa a, un moment, envisagé de se convertir au protestantisme et a, pour un temps, appuyé le grand projet avorté de Tavares Bastos (1862) de faire venir des États-Unis, en raison de la Guerre de Sécession (1861-1865), un demi-million de "protestants éduqués ». Cette articulation protestantisme/franc-maçonnerie visait à trouver des solutions à ce qui apparaissait alors aux yeux des élites comme le péril le plus grand, à savoir l'énorme poids démographique et culturel des populations noires et mulâtres dans un pays qui, alors, se représentait idéalement comme blanc et européen.

\section{2 - Cultes afro-brésiliens et spiritisme kardéciste}

À Recife justement, au tournant du siècle, la population afro-brésilienne représentait plus de $60 \%$ des habitants de la ville. Les expressions religieuses les plus visibles de ces populations passaient par le canal du catholicisme qu'on leur avait imposé. Mais René Ribeiro (1978, p. 28) montre bien que, déjà vers 1640, on peut clairement identifier sur une gravure du Zoobiblion du Hollandais Zacharias Wagner, une danse dont tous les éléments correspondent à la roda de xangô telle qu'on a pu l'observer au $X^{\mathrm{e}}$ siècle. D'autres références écrites se trouvent dans des documents datant du XVIII', mais ce n'est qu'à partir de la seconde moitié du $\mathrm{XIX}^{\mathrm{e}}$ qu'on peut retrouver la trace et l'emplacement de ces lieux de culte, qui se sont développés surtout après l'abolition de l'esclavage, avec l'arrivée d'une masse rurale qui se retrouvait abandonnée à elle-même dans un milieu urbain, et dont l'un au moins existe encore aujourd'hui (Sitio do Pai Adão) ${ }^{9}$.

Les chefs de culte étaient alors soit d'anciens esclaves domestiques qui connaissaient bien la ville, soit des membres de familles africaines arrivées après l'abolition de l'esclavage. Ceux-là venaient des côtes de l'actuel Bénin et du Nigeria où se trouvaient beaucoup d'anciens déportés de la Révolte des Malês de $1835^{10}$. Ils ont ainsi contribué à asseoir la prépondérance de leur panthéon nagô ce qu'il est aujourd'hui - dans les emprunts et les mélanges qui se sont produits entre les diverses ethnies africaines établies au Brésil. Quelle que soit la «nation» à laquelle ils se rattachaient, tous prônaient un lien intrinsèque entre l'homme et la nature; leurs divinités sont les émanations d'un mélange d'éléments naturels et culturels. Jusqu'à présent, dans les terreiros (lieux de culte) «traditionnels» (xangôs) l'élément central de l'assentamento (la représentation matérielle de la force dynamique de la divinité) est une pierre (otá) ${ }^{11}$.

\footnotetext{
${ }^{9}$ Ce lieu de culte a été, en 1982 , décrété «monument historique » par l'État du Pernambuco.

10 Plusieurs des leaders de cette révolte avaient été déportés en retour vers l'Afrique et la présence de ces «Brésiliens » fomenta un intérêt certain pour l'autre côté de l'Atlantique de la part de familles africaines dont quelques-unes firent librement la traversée.

${ }^{11}$ Pour plus de détails sur ce sujet cf. Marion AubréE, 1985, pp. 187-199.
} 
Au début du siècle, les spirites étaient peu nombreux et très discrets à Recife. Leurs rituels étaient d'ordre privé et les fédérations qu'on connaît aujourd'hui n'avaient pas encore pignon sur rue. Ils jouissaient de la sympathie des francs-maçons car ils partageaient avec eux la foi dans le progrès, dans lequel ils voyaient le moteur des transformations sociales, un désir de fraternisation entre toutes les classes et les races ; comme eux, ils voyaient le salut dans le perfectionnement individuel et, comme eux, ils avaient eu à souffrir des attaques de l'Église catholique.

À l'inverse, les cultes afro-brésiliens étaient assez nombreux et regroupés dans le nord-ouest de la ville (Beberibe, Aguas Frias, Casa Amarela) là où se trouvait la population noire la moins métissée. Leurs rituels, rythmés par le battement des tambours sacrés, ne pouvaient pas passer inaperçus. Face au monothéisme chrétien triomphant, les élites et les classes montantes considéraient que le polythéisme avéré des cultes de type traditionnel était porteur d'une vision archaïque du monde qui devait dans la mesure du possible être éradiquée ${ }^{12}$.

Le caractère négatif du jugement porté par ces élites sur les cultes afro-brésiliens est très bien documenté à ce jour. En effet, l'esprit d'ordre et de progrès de l'époque a amené, en particulier et comme nous l'avons vu, les médecins psychiatres à s'intéresser aux pratiques religieuses afro-brésiliennes pour des raisons $\mathrm{d}^{\prime}$ hygiène mentale ${ }^{13}$. Ce souci " eugéniste », non dénué de préjugés, a incité ces médecins à étudier ces populations de près et à produire des monographies qui, une fois débarrassées de certaines réflexions fortement teintées de positivisme et d'évolutionnisme, fournissent des descriptions minutieuses concernant les rituels, les liens sociaux, la hiérarchie et le fonctionnement de ces groupes socio-religieux.

Dans les années 1930, malgré les préventions évoquées ci-dessus, les cultes afro-brésiliens étaient très vivaces à Recife - même si la plupart de leurs membres participaient également aux cérémonies de l'Église catholique -, comme en témoignent les travaux des jeunes psychiatres qui participaient à la Ligue d'Hygiène Mentale mentionnée plus haut et qui, à ce titre, visitaient régulièrement les lieux de culte ${ }^{14}$ des quartiers pauvres, peuplés de gens dont la plupart étaient descendants d'esclaves (Beberibe, Fundão, etc.).

Ainsi, dans son ouvrage Xangôs do Nordeste, Gonçalves Fernandes nous fournit des informations précieuses sur divers terreiros, sur les rituels qui y étaient pratiqués, sur les principaux chefs de culte et leurs relations avec les autorités civiles et sanitaires ainsi que sur les propositions émises par les uns ou les autres quant à la « bonne » façon de pratiquer leur religion et, implicitement, d'acquérir une légitimité aux yeux des classes dominantes.

Certains observateurs ne voyaient pas d'un œil très amène les expressions religieuses afro-brésiliennes et, dans l'introduction à son ouvrage Xangô (1937), Vicente Lima souligne qu'il s'agit pour lui d'analyser "l'un des plus tristes aspects de la scène sociale de (sa) terre et de (son) peuple».

\footnotetext{
12 Signalons que cette volonté d'éradication n'a pas eu d'effets à ce jour puisque, malgré diverses dynamiques impulsées jusque dans les années 1960 par les classes moyennes pour en venir à bout, les cultes polythéistes de type traditionnel sont plus vivants que jamais au cœur de la société brésilienne.

${ }^{13}$ Cf., entre autres, pour le Nordeste les travaux de Raymundo Nina Rodrigues, 1900, Pedro Cavalcanti, 1933, Arthur Ramos, 1934, Gonçalves Fernandes, 1937.

${ }^{14}$ Cf. la description des divers cultes faite par Pedro CAVAlCANTi, op. cit., pp. 58-63.
} 
Pourtant, après avoir décrié sur six chapitres ce qu'il appelle la « liturgie fétichiste », ce même auteur envisage la disparition des cultes afro-brésiliens au profit du panthéisme tel qu'il se présente dans la vision du monde et les pratiques rituelles du groupe qui nous intéresse ici "Círculo Deus e Verdade » - dont je vais décrire les caractéristiques ci-après - ; il y voit la nécessaire "évolution religieuse du noir brésilien ».

\section{Fondation et croissance du Cercle Dieu et Vérité}

L'association «Circulo Deus e Verdade » fut créée le 2 juillet 1929 au 910 de la Estrada de Beberibe par José Amaro Feliciano dont on nous dit qu'il était un "homme intéressant» qui savait lire et écrire (Cavalcanti), "mulâtre grand et maigre à l'allure austère » (Valente). Par ailleurs, certains auteurs signalent que le «Prof. Amaro " avait été formé à l'école de Bento Milagroso, un "prophète » ${ }^{15}$ apparu dans ces mêmes quartiers pauvres de Recife en 1915. Celui-ci ne se réclamait pas du panthéisme mais il soignait les malades avec de l'eau selon une tradition que l'on retrouve jusqu'à ce jour dans les cultes spécifiques de la région que sont la jurema et le catimbó ${ }^{16}$. Nous allons voir que dans le Cercle cette importance symbolique de l'eau allait être conservée et élargie.

L'association, dont le temple a été rapidement installé dans le quartier du Fundão, l'un de ceux qui regroupait comme on l'a vu de nombreux terreiros, eut une durée de vie relativement longue puisqu'en 1964, selon W. Valente, elle existait toujours malgré les persécutions de l'Estado Novo, le déménagement dans le quartier d'Iputinga et la mort récente de son fondateur. Elle avait, par ailleurs, obtenu une personnalité juridique le 28 février 1935 sous le nom de Circulo Panteista Deus e Verdade, ce qui lui donnait une reconnaissance sociale certaine. Le fondateur était secondé par un directoire composé de 24 definidores, appelés ainsi parce qu'ils participaient à la définition de la doctrine. Celle-ci était, dans un premier temps, basée sur l'adoration des planètes Mars, Neptune, Jupiter et Uranus, représentées de façon anthropomorphique dans le temple par des têtes de plâtre, et de l'étoile Vestra ${ }^{17}$ qui trônait au fond du sanctuaire au-dessus d'un bassin plein d'eau et était surmontée d'un arc-en-ciel, l'ensemble représentant le ciel, la terre et

${ }^{15}$ Ce terme est ici employé au sens classique où il est entendu dans le Nordeste brésilien depuis le milieu du XIX ${ }^{\mathrm{e}}$ siècle, c'est-à-dire d'homme de foi (catholicisme populaire) qui réunit les foules en leur apportant un bien-être spirituel ou physique (prêche et miracles). Parmi les « prophètes » les plus célèbres on compte Antonio Conselheiro (Canudos) et Padre Cícero (Juazeiro do Norte), ou encore Frei Damião mort récemment.

16 Pour plus de détails sur ces deux cultes voir Roberto MotTA, 1988, pp. 27-48.

${ }^{17}$ Il existe un astéroïde du nom de Vesta mais il n'a pas été possible de savoir s'il est à l'origine du nouveau nom. Le seul élément proche phonétiquement serait l'adjectif latin vestras : ce qui est de votre famille, de votre pays (GAFFIOT). Certes Feliciano, maçon de son état, ne devait pas connaître cette langue mais on peut suggérer que, de formation catholique, il l'avait peut-être entendu lors d'une messe en latin et l'ayant trouvé agréable à l'oreille en a fait le centre de son univers cosmologique. En effet, on connaît bien des cas, au Brésil, où les noms sont des adaptations phonétiques d'un mot ou d'un nom originaire d'une autre langue. Par ailleurs, on note à plusieurs reprises dans les textes des observateurs des références à l'adoration de l'Étoile Blanche (Vénus). 
leur union (Lima, p. 59). Sur le plan terrestre J. A. Feliciano donnait une importance toute particulière, dans sa doctrine et les rituels structurés autour de celle-ci, à l'eau vive et aux grandes roches. Il est intéressant ici de constater qu'à ses débuts, dans cette ville installée en bord de mer, le Cercle se tournait plutôt vers l'intérieur du pays pour mettre au centre de l'adoration de ses disciples [plusieurs centaines selon tous les auteurs] les sources et les rivières ainsi que les monticules rocheux qui émergeaient de la terre. À partir de ce dernier élément W. Valente parle de litholâtrie, dont il est aussi question chez plusieurs auteurs à propos des cultes afro-brésiliens. Il rattache cette tradition à des coutumes amérindiennes ainsi qu'à d'autres mouvements socioreligieux qui ont surgi dans le sertão de Pernambuco au cours du XIX X siècle $^{18}$.

\section{1 - Les années trente}

Le temple, les cérémonies et activités qui y avaient lieu ont été décrits et analysés, d'une part, pour le début des années trente par Pedro Cavalcanti et Vicente Lima et, d'autre part, pour le début des années soixante par Waldemar Valente. Dans le premier cas, Cavalcanti remarque la profusion de lumière électrique ${ }^{19}$ qui illumine le temple et l'architecture «bizarre » qui attire l'attention. Sur la photographie qui présente l'extérieur du temple on remarque une façade concave en trois parties dont les lignes et la décoration rappellent un peu les productions de l'École du Bauhaus ; elle devait, à ce moment-là, être d'une grande originalité par rapport aux constructions environnantes. À l'intérieur, l'espace central est entouré par des sortes de "balcons" qui servent à séparer les hommes des femmes et les fidèles des visiteurs; deux autres balcons sont réservés l'un aux membres du directoire et l'autre à l'autel sur lequel reposent des têtes de plâtre représentant les quatre planètes sus-mentionnées ainsi que d'innombrables verres remplis d'eau (Cavalcanti, p. 59).

En outre, un espace particulier est réservé à la «table» sur laquelle prennent place des objets, en terre cuite selon Cavalcanti, en sable mouillé selon Lima, faits par les adeptes du Cercle à la veille des cérémonies. Si Cavalcanti a vu là des chaînes de montagnes, des champs et des vallées, Lima, lui, a observé des représentations de "sphinx » qu'il n'hésite pas à attribuer à un "transport d'inspiration religieuse des Égyptiens » obtenu à travers les communications spirituelles reçues durant les états médiumniques.

Les cérémonies au siège avaient lieu à partir de 19 heures et la première heure « de travail » était consacrée à l'adoration des planètes; elles se prolongeaient ensuite par des enseignements sur la doctrine ; elles avaient lieu les mardis, jeudis et dimanches. En entrant dans le temple chacun revêt sa blouse blanche ${ }^{20}$, sur laquelle est brodé en bleu l'emblème du Cercle. Une jeune fille, considérée comme

18 Cité du Paradis Terrestre de la Serra do Rodeador (vers 1820) et Royaume Enchanté de Pedra Bonita (années 1830).

19 À cette époque les maisons modestes ne bénéficiaient pas de l'électricité d'où, sans doute, la remarque de Cavalcanti.

${ }^{20}$ Ces vêtements blancs et l'abstention d'alcool seraient, selon G. FreYre (op. cit.), le signe d'une influence musulmane dans la mesure où un grand nombre de noirs arrivés en tant qu'esclaves dans la région étaient islamisés. 
porteuse de pouvoirs spéciaux, entonne les prières et les chants. La même se place devant l'autel et commence à balancer la tête d'un côté à l'autre, ce qu'elle fera pendant toute l'heure tout en déclamant des vers et chantant des cantiques qui parlent des eaux, des forêts, de la lune et des planètes. Pedro Cavalcanti remarque que la musique est monotone et pauvre ; elle rappelle, selon lui, celle des temples protestants alors présents dans le paysage religieux de Recife. Même si l'auteur n'y fait qu'une brève référence à la fin de son article par rapport aux enfants, on peut induire de sa description que la jeune fille entre ainsi en transe de possession pour recevoir «l'Habitant de la planète » qui lui dicte le texte des cantiques. On comprend qu'il s'agit d'un esprit venu d'ailleurs. Mais il n'existe pas dans ce texte d'éléments plus explicites sur cette entité. Durant cette séquence, des enfants des deux sexes se joignent à la prêtresse autour de l'autel et entrent, eux aussi, dans un état altéré de conscience par la mise en mouvements non seulement de la tête mais de tout le corps; ce que G. Freyre qualifie de "danse ». Selon les " adorateurs de la nature » eux-mêmes, ils sont alors «irradiés » par la même entité venue de la planète adorée à ce moment-là. Chaque 15 minutes une clochette "électrique » interrompt les mouvements et oblige au silence pour quelques instants. On ne sait si cette coupure correspond à un changement de "planète ».

Cavalcanti souligne un détail intéressant; pendant la transe, la jeune fille et les enfants dialoguent dans une "langue étrange ... absolument improvisée ». Cela nous ramène aux phénomènes de glossolalie ${ }^{21}$ qu'on observe aujourd'hui plus particulièrement dans les groupes pentecôtistes et qu'on attribue généralement à une origine nord-américaine. Or, le premier groupe pentecôtiste d'origine états-unienne, l'Assemblée de Dieu, n'est arrivé à Recife qu'au début des années trente. On peut donc supposer que dans cette société nordestine où beaucoup de gens n'avaient pas la maîtrise du bien-parler, les messages ésotériques obtenus durant la transe étaient souvent l'une des formes d'expression publique ou, au moins, communautaire des personnes qui se trouvaient au bas de l'échelle sociale.

On retrouve également ici l'importance de la médiation féminine qui apparaît dans presque tous les mouvements socioreligieux surgis dans le Nordeste du Brésil entre 1820 et $1938^{22}$. Par ailleurs, toutes les semaines avait lieu une distribution d'eau « irradiée » qui, tout comme l'eau bénite des catholiques, avait à la fois des vertus de protection domestique et des vertus thérapeutiques contre les petits maux quotidiens; ainsi chacun en buvait le matin pour se maintenir en bonne santé. On apprend par Cavalcanti qu'en 1933 J. A. Feliciano, suite au succès de sa doctrine à Recife, est parti pour l'Etat du Pará en Amazonie afin d'y fonder un autre Cercle suivant ses enseignements. Je n'ai pu, à ce jour, retrouver la trace de cette filiale amazonienne. Enfin, parmi les activités non rituelles, Cavalcanti signale que cette communauté maintenait une école où les adeptes et les enfants apprenaient à lire et à écrire ainsi qu'un cours de "catéchisme ", sans préciser s'il s'agissait là de l'enseignement de la doctrine panthéiste ou du catéchisme catholique ${ }^{23}$.

${ }^{21}$ Cf. Marion AubréE, Paris, 1996, pp. 173-192.

22 Voir sur le sujet Marion Aubrée, 1997.

${ }^{23}$ Ceci serait toutefois surprenant dans la mesure où les « adorateurs des planètes » disent, selon le même auteur, n'avoir pas foi dans le Christ puisqu'il est venu sur terre pour souffrir et que leur religion, résolument optimiste, rejette la souffrance. 
Par ailleurs, les rituels autour de l'eau vive engendraient des déplacements en groupe, loin de la ville. Un des auteurs (Lima) a participé à l'un d'entre eux et a pu le décrire. Il s'agit d'une retraite à la Cascade de Pilões (intérieur du Pernambouc) à environ trois heures de train de la capitale. Elle eut lieu le 15 novembre 1936 et réunit 200 panthéistes (hommes, femmes et enfants). Il note qu'à leur arrivée, les membres du groupe prirent une collation dans une école publique où une " gentille professeur" les accueillit "malgré la censure locale représentée par les catholiques et par le curé de l'endroit» (op. cit., p. 64). Ils se dirigèrent ensuite vers le lieu de l'adoration où le Penseur (J. A. Feliciano) donna, à midi, le signal du recueillement en levant la tête vers le firmament. Là encore, c'est une jeune fille qui reçoit l'Habitant (de la planète) et rapidement la transe devient contagieuse, au point que tous se précipitent dans ce «Jourdain pernamboucain » puis se laissent glisser dans les cascades " complètement transfigurés et parlant des langues étranges » (p. 65). Le Directoire panthéiste assiste à la scène en se tenant les mains et dans le plus complet silence. L'ensemble du culte a duré deux heures et pour le clore on fait tinter la clochette. Après quoi on sert un repas «naturiste ». À dix-huit heures, l'adoration reprend au moment où apparaît Vénus dans le crépuscule. Les adorateurs, nous dit Lima, l'appellent Jupi, sans que l'on ait plus d'explications sur cette appellation; ils disent également qu'elle fut amenée là par Helios. Le groupe lui attribue aussi d'autres noms tels que : Étoile de la Nuit, Étoile des Prophètes, Étoile des Panthéistes. Lorsqu'elle s'élève dans le ciel, le Penseur entonne un hymne d'hommage : Deus vos salve a Luz da Lua / Que do céu nos avistou / Deus vos salve a estrêla / Que as aves visitou; ce qui donne, en traduction libre : Dieu te sauve Lumière de la Lune / Qui nous a vus depuis le ciel / Dieu te sauve ô étoile / Que les oiseaux vont visiter.

La description de Lima continue avec le surgissement de la pleine lune et l'apparition des constellations qui «bordent le ciel ». Cette nouvelle phase d'adoration se prolonge jusqu'à vingt heures puis tous se reposent. À minuit, Feliciano salue ce moment-clé et la nature qui les entoure. C'est alors que l'Habitant réapparait ${ }^{24}$ et commence à « irradier ». Le Penseur le salue tandis que d'autres Habitants se manifestent, dans le corps de trois jeunes femmes, dont l'un d'eux s'adresse à la foule dans les termes suivants :

"Que Dieu vous sauve tous - Enfants, que dans cette nuit bénie vous communiiez dans cette touchante réunion de confiance religieuse » (ils ne parlent jamais de foi, précise Lima). Suivent deux paragraphes d'exhortation à la confiance et à la construction de l'édifice moral, dans la joie de l'existence (ils sont, comme nous l'avons vu, optimistes et rejettent la souffrance). Le discours s'achève sur un cri : «Panthéistes, soyez forts! Par votre volonté vous triompherez du mal. Dites dans toutes les circonstances de votre existence: J'ai été, je suis et je serai panthéiste .....Alá pama ainaura, Cassarunei qui lá nura, Sonoquliqui, Clecissi lá nura » (que Lima consigne comme "langue étrange » et que nous pouvons considérer comme une forme glossolale susceptible d'être comparée à quelques productions actuelles de groupes religieux de confession bien différente). Le fondateur répond à ce salut

${ }^{24}$ Le texte suggère que, cette fois, il ne vient pas d'une planète mais de la forêt environnante car Lima fait allusion à une étude qu'il fait parallèlement : "Morada de habitante " in Estudos sobre o Panteismo qui, en définitive, n'a jamais été publiée. Il pourrait donc s'agir de la résurgence de figures symboliques amérindiennes, liées à la vie de la forêt. 
tandis que les fidèles pleurent sur ceux qui se sont éloignés de la doctrine ou qui sont morts. Pour sa part, l'auteur commente qu'il n'a " jamais vu tant d'ordre et de conviction religieuse ».

L'enthousiasme de V. Lima face à cette "élite d'hommes prolétaires, sains et forts, honnêtes et travailleurs » (p. 61) est sans bornes. Il y reconnaît une dimension franc-maçonne (obédience dont lui-même se réclamait) qui lui fait entrevoir un avenir radieux pour le peuple de sa région. D'autant que la doctrine sociale du Cercle va dans le sens de sa propre vision du monde puisqu'on y travaille sans individualisme et pour le bénéfice de tous ; on y condamne l'exploitation financière et on y interdit l'entrée d'argent, si ce n'est pour la réalisation matérielle d'actions au bénéfice du patrimoine social, c'est-à-dire des dons pour améliorer les installations communautaires. Enfin tout, chez les panthéistes "obéit à un rythme évolué », où les hymnes dédiés au Dieu-Nature contrastent totalement avec les chants "horribles » des cultes afro-brésiliens qu'accompagne le rythme des tambours.

Il faut ajouter que de telles retraites avaient aussi lieu vers des monticules rocheux, comme nous pouvons le constater à partir d'une des photographies présentées par Lima au Monte de Giz d'Olinda ; ceux-ci donnaient également lieu à des rituels d'adoration de la Nature qu'aucun des auteurs n'a décrit dans le détail. En outre, des cérémonies propitiatoires étaient menées par les femmes, et par elles seulement, le long des fleuves où elles pratiquaient un semblant de pêche dont le but était de stimuler la Nature, dans sa forme fleuve, afin que celui-ci offre le poisson en quantité.

\section{2 - Les années soixante}

Trente ans après la fondation, les symboles et les rituels apparaissent quelque peu différents dans l'exposé qu'en fait W. Valente ; ils nous permettent de voir la dynamique de transformation à l'œuvre dans ce groupe devenu, officiellement, le «Cercle panthéiste Dieu et Vérité ». À cette époque J. A. Feliciano donne à Valente sa propre étymologie de «panteistas » (pan = Dieu et istas = amis de Dieu et des royaumes »); ce qui fait dire à l'observateur que Feliciano " a entendu le coq chanter mais pas très bien précisé d'où cela venait », locution communément employée pour dire d'une personne qu'elle n'a qu'une vague notion de ce dont elle parle ou qu'elle a recréé cette notion à partir de bribes de connaissances. Toutefois le leader précise encore plus sa pensée quand il dit «Dieu-Nature parce que Dieu c'est le masculin et la Nature c'est le féminin ».

C'est en 1946, après les persécutions varguistes, dont les cultes afro-brésiliens furent eux aussi la cible ${ }^{25}$, que Feliciano put en toute légalité construire un nouveau temple dans le quartier d'Iputinga. Celui-ci était, aux dires de Valente, «d'un style encore plus surréaliste que le premier »; la photographie qui accompagne le texte ne nous permet pas de déterminer cette dimension surréaliste. Il nous signale

${ }^{25}$ Ainsi, un de mes informateurs, lui-même chef de culte, me disait au début des années quatre-vingt que le temple de tradition jejê de sa mère (-de-saint) avait été détruit par la police au début des années quarante mais que tous les objets sacrés communautaires avaient été récupérés par Gilberto Freyre et qu'ils figuraient dans les collections du Musée de l'Homme du Nordeste de la Fondation Joaquim Nabuco. 
cependant, en tant que psychiatre, qu'il est le fruit d'une "imagination peut-être schizö̈de ou paranoïde » (p. 161). Il ajoute qu'il s'agit d'un panthéisme rigoureusement nordestin, "intégré aux conditions écologiques ; subordonné aux influences du milieu physique et du milieu culturel».

L'influence du milieu culturel se mesure au fait que la doctrine s'est transformée, au moins quant à ses figures de référence. Les représentations des planètes ne sont plus centrales dans le temple ; elles ont été supplantées par des personnages du catholicisme populaire tels São Severino dos Ramos (habillé en militaire), le Padre Cícero ou Dom Vital, l'évêque anti-maçonnique dont il a été question plus haut. On y trouve aussi le buste du poète portugais Camões qui célébra de façon si lyrique la culture lusitanienne. L'arc-en-ciel a été remplacé par l'œil sans paupière, symbole de l'Essence et de la Connaissance, qu'on peut attribuer à la fois à la tradition chrétienne et à la tradition maçonnique et qu'on retrouve actuellement dans quelques temples pentecôtistes à Recife. D'autre part, le Penseur-fondateur n'est plus, durant les cérémonies, au milieu du directoire, il se tient sur un "trône » situé à l'une des extrémités de la salle, devant un bassin avec jets d'eau, au-dessus duquel apparaît toujours l'étoile Vestra. Il fait face à un autel dédié au Padre Cícero qui est lui-même flanqué d'une horloge peinte en forme de visage aux yeux ouverts qui symbolise le temps. On avait déjà vu que les cérémonies étaient précédemment soumises à une certaine ponctualité, se rapprochant en cela de la tradition de régularité du spiritisme kardéciste dont, cependant, J. A. Feliciano a toujours affirmé n'être en rien tributaire, de même qu'il rejetait une quelconque filiation avec les cultes afro-brésiliens.

Par ailleurs, si les definidores existent toujours, leur uniforme blanc et bleu ne sert plus que pour de grandes cérémonies. Le reste du temps il est remplacé par un uniforme gris qui ressemble à un complet veston. La majorité des adeptes sont qualifiés non plus d'adorateurs mais de romeiros (pèlerins), terme employé pour désigner ceux qui se rendent dans les sanctuaires de dévotion du catholicisme populaire brésilien. Ceux-là sont toujours habillés de blanc avec un détail bleu. On trouve aussi dans le texte de Valente le terme «initiés", sans qu'il soit précisé à aucun moment à qui exactement on se réfère et en quoi consisterait l'initiation. Au niveau de la hiérarchie, on distingue parmi les femmes les "catéchistes », qui enseignent aux enfants, nombreux, les rudiments de la doctrine, et les « jardinières » qui cultivent les fleurs, source de parfum et de beauté, dont les premiers observateurs n'avaient pas parlé. Par ailleurs, W. Valente repère dans le Cercle nouveau des traces de sébastianisme. Cette croyance dans le retour du roi Sébastien, tué à la bataille d'Alcacér le 4 août 1578, avait été importée du Portugal et resta très présente dans l'imaginaire nordestin durant tout le XIX ${ }^{\mathrm{e}}$ siècle ${ }^{26}$. Aujourd'hui encore, des traces de ce millénarisme resurgissent sporadiquement dans des lieux de culte inattendus tels certains terreiros afro-brésiliens de São Luis do Maranhão ${ }^{27}$.

${ }^{26}$ Au sujet de ce mythe et de sa diffusion au Portugal voir L. VALEnSI, 1992. Par rapport à la diffusion du mythe au Brésil Valensi signale qu'elle avait été frappée, en lisant Os Sertões d'Euclydes da Cunha par le fait que les insurgés chantaient le retour attendu du roi Sébastien. De même, les deux mouvements socioreligieux dont il est fait mention dans la note 18 étaient très marqués de sébastianisme.

27 Cf. Mundicarmo Ferretti, 1994, p. 58. 
Par ailleurs, les retraites vers les sources de l'intérieur continuent alors de drainer beaucoup de monde ( 800 personnes pour celles du fleuve Gurjau au début des années cinquante selon un reportage du Diario da Noite) mais les photographies font apparaître que les fidèles vont aussi rendre hommage à la mer, toute proche mais dont il n'avait pas été question précédemment.

Enfin, l'iconographie nous permet d'observer deux cérémonies autour de la naissance d'un enfant; elles ont pour but de protéger le nouveau-né. Dans la mesure où les photos permettent d'en juger, le public semble essentiellement féminin et noir. L'enfant est étendu au sol sur un matelas et entouré de divers objets symboliques dont certains sont directement issus de la nature (sable, tronc d'arbre, fruits, poissons) et d'autres ont une portée plus culturelle (plats, monnaie d'or, buste de femme, tableau représentant un œil énorme et, aussi, un grand serpent artificiel). Je n'ai, à ce jour, pas trouvé d'informations me permettant de donner leur juste signification à ces objets dans le cadre de cette communauté. On peut simplement inférer que la présence de l'or est un vœu de prospérité, que l'œil symbolise le désir d'ouverture de l'enfant vers la Connaissance prônée dans le groupe et que le serpent s'est, peut-être, substitué à l'arc-en-ciel comme symbole de relation entre la terre et le firmament qui sont les supports entrecroisés et indissociables de la cosmologie des panthéistes que nous venons de suivre.

\section{Bricolage, syncrétisme et métissage}

Ni animisme, ni naturisme (au sens de Max Muller), le Cercle Panthéiste Dieu et Vérité a fourni à des milliers de personnes une structure symbolique et une communauté de croyance qui les ont aidées à s'inscrire, à la fois dans la société qui les englobait, grâce à la liaison qu'il assurait avec l'idée nationale dont le mode de pensée et les valeurs étaient diffusés par les élites, et dans un continuum historique qu'on peut qualifier de « populaire », c'est-à-dire partagé par le plus grand nombre, grâce à la refonte d'éléments mythiques et mystiques à laquelle il procédait, éléments qui, au Brésil, semblent être en mesure de survivre à tous les changements de conjonctures socioculturelles et politiques.

Pour résumer on peut dire que dans le Cercle Panthéiste Dieu et Vérité on pratiquait un culte résolument positif dans la mesure où les rituels étaient, sans exception, des rituels d'adoration ou des rituels propitiatoires; les notions de sacrifice, d'expiation, de repentir ou de pénitence sont, au vu des éléments qui ont été réunis à ce jour, totalement absents de cette doctrine, tandis que certains rituels donnent lieu à une dynamique d'adorcisme collectif. Par ailleurs et quant à la doctrine, sur une période de trente ans, on observe un glissement du naturalisme vers le déisme, lequel prévaut dans les explications fournies à Valente par Feliciano au début des années soixante. En ce qui concerne l'agencement du temple, les planètes ont été mises en retrait, au profit de figures historiques issues de la pensée luso-tropicale alors dominante. Avec la complexification de la doctrine et, peut-être aussi la dynamique de différenciation sociale qui s'opère au sein du groupe, la hiérarchie interne crée de nouveaux statuts, en particulier pour les femmes. 
Notons encore que si Bento Milagroso était qualifié de "prophète», J. A. Feliciano, lui, est désigné par les termes de " professor» ou de "mestre »; cette dernière appellation viendrait confirmer une influence amérindienne puisqu'elle sert généralement, dans le contexte socioreligieux, à désigner le pajé, mot d'origine tupi-guarani qui signifie sorcier-guérisseur. Toutefois, il convient de souligner que, dans ce cas, on n'a remarqué aucune interaction thérapeutique entre le penseur-fondateur et ses fidèles; seule l'ingestion d'eau "irradiée » peut être considérée comme telle, et elle procède du rituel collectif. Le couplage des deux termes semble indiquer l'importance que prend une référence moderne sans que soit abandonnée la référence traditionnelle. En effet, à l'époque, le terme de «professeur » était attribué à ceux qui - hommes et femmes - avaient reçu une formation et un diplôme pour l'enseignement du premier degré et, par extension, à des personnes considérées comme dépositaires d'une connaissance hors du commun.

C'est donc dans les rituels qu'on note la plus forte continuité, notamment dans l'importance accordée à l'adoration en groupe de tout ce qui représente la Nature, dans laquelle les grandes pierres et l'eau sous toutes ses formes sont centrales mais aussi, nous dit Valente, les plantes, les fleurs, les fruits, les animaux, les montagnes, la terre, les vents et les astres. Ce sont ces rituels qui déterminent les «formes de l'expression du panthéisme créé à Recife par l'imagination mystique de José Amaro Feliciano » (Valente, p. 171).

Dans cette «expression » on peut reconnaître, même si Feliciano les rejettent, des similitudes avec ce qu'on observe à la fois dans le spiritisme kardéciste (ponctualité, morale sociale), cette religion des classes moyennes considérée comme une tendance vers ce qui était qualifié de "progressiste », ainsi qu'avec ce qui faisait alors la spécificité des cultes afro-brésiliens, à savoir la transe de possession et l'importance symbolique de la pierre; le ressourcement par le contact avec l'eau et les pierres et l'apparition sporadique "d'esprits de la forêt » nous renvoient à des traditions amérindiennes qui n'ont jamais disparu, même dans l'imaginaire des citadins. Par ailleurs, on trouve des symboles maçonniques et chrétiens plus généraux. Tous ces éléments divers structurés en un véritable "système religieux ", au sens de Durkheim, permettent d'explorer une voie d'analyse qui aide à mieux comprendre l'attrait qu'a exercé un arrangement symbolique de ce type auprès des milliers de gens qui y ont adhéré au cours de ses quarante années de vie.

Les notions de bricolage, syncrétisme et métissage évoquées en-tête de cette section pourraient probablement toutes trois s'appliquer, dans une certaine mesure, au cas particulier qui nous intéresse ici. En effet, si on reprend la métaphore développée par Lévi-Strauss dans La Pensée Sauvage, on voit que, comme le bricoleur, le Penseur avait à sa disposition un ensemble hétéroclite, résultat contingent de toutes les occasions qui se sont présentées à lui d'enrichir son stock de matériaux symboliques ${ }^{28}$ afin de réaliser son projet. On a pu également constater qu'il n'a pas hésité, au cours des ans, à déplacer certaines figures premières (les planètes) en faveur d'autres qui s'inscrivaient plus directement dans la dynamique générale de la société englobante (saints catholiques, Padre Cícero) ou qui pouvaient lui apporter un surplus de légitimité sociale (Camões ou Dom Vital).

\footnotetext{
${ }^{28}$ LÉVI-STRAUSS, 1962, pp. 26-33.
} 
Toutefois, on l'a vu, ce stock n'était pas seulement alimenté par deux « ensembles matriciels »- notion qui a servi de fondement à l'une des dernières analyses en profondeur du fait syncrétique ${ }^{29}$ - même si c'est le plus souvent le cas dans les situations de colonisation et l'acculturation subséquente. Bastide déjà avertissait : «le terme de syncrétisme a peut-être le tort de cacher, en les dissimulant sous l'identité d'un même terme, toute une série de phénomènes ou de processus extrêmement différents ${ }^{30}$. Dans le cas du Brésil, en effet, il semble bien difficile de faire des analyses binaires. Comme le signale P. Sanchis dans un article datant de 1994 (pp. 4-11), dans cette culture, il existe toujours un tiers, culturel ou autre, susceptible de venir bousculer les logiques d'oppositions et de créer une dynamique nouvelle. Enfin, contrairement à ce qui ressort de la vision lévi-straussienne du bricolage, il me semble qu'il y a bien là une "création de matière » dans la mesure où, s'agissant d'une innovation religieuse, Feliciano voulait réduire au minimum les ambivalences en bouchant les trous qui auraient pu affecter la logique interne de son système.

Ainsi, ce projet contient des éléments véritablement nouveaux; il n'y a pas eu seulement agencement mais bien création d'un univers original à partir d'une situation où il fallait se démarquer des autres et tracer des "frontières" - au sens où Fredrik Barth entend celles-ci dans son analyse des groupes ethniques - pour créer de l'identité. On peut ainsi suggérer que, dès cette époque, mais c'est encore très souvent le cas aujourd'hui au Brésil, c'est le référent religieux plus que le référent ethnique qui sert à construire des identités différenciées ${ }^{31}$. De fait, pour des populations qui ont toujours vécu dans le brassage ethnique et le métissage culturel, il était important de trouver des éléments susceptibles de fixer, au moins pour un temps, une identité fondée sur des rituels spécifiques, sur la croyance partagée en des figures emblématiques et des valeurs socialement acceptées, agencés selon un ensemble de signes diacritiques permettant aux adeptes de se distinguer de ceux qui les entouraient. En effet si, comme l'affirment F. Laplantine et A. Nouss ${ }^{32}$, le métissage est une «pensée de la tension », on observe que ceux qui le vivent - au moins dans les classes les plus modestes - ont toujours cherché, et ce jusqu'à présent, à soulager cette tension en découpant des pans de la réalité symbolique et matérielle afin de se créer un horizon prévisible qui les aide à se stabiliser.

Je terminerai en soulignant le fait que le Cercle Panthéiste Deus e Verdade, mouvement socioreligieux urbain créé par un leader issu d'un milieu modeste, n'a survécu que de peu à la disparition de son fondateur. Pourtant sa doctrine contenait déjà les germes d'une pensée écologique qui s'est, depuis, diffusée au Brésil sous des formes plus hédonistes ou plus mystiques. Ce mouvement a également été, dans une certaine mesure, le précurseur des grands rassemblements d'hommage à la déesse de la mer afro-brésilienne, Iemanjá ${ }^{33}$. On peut enfin relever des similitudes

${ }^{29}$ Cf. André MARY : Le défi du syncrétisme Paris, Éditions de l'EHESS, 1999 (cf. Arch. 110-35).

${ }^{30}$ Voir, en particulier, son ouvrage Le prochain et le lointain, 1970, p. 237.

31 Par rapport à cette problématique de construction ou reconstruction identitaire à travers l'appartenance à une communauté religieuse, cf., Marion AubréE, op. cit., 1985.

32 Cf. F. Laplantine, A. Nouss, 1997.

${ }^{33}$ Ceux-ci ont lieu tous les ans dans la nuit de la Saint-Sylvestre et réunissent des millions de gens sur le littoral atlantique. 
entre ce groupe religieux et la religion du Santo Daime ${ }^{34}$, créée en Haute Amazonie dans les années trente et dans laquelle on trouve des éléments d'organisation sociale (étapes hiérarchiques marquées par des vêtements particuliers, séparation hommes/femmes) et des expressions symboliques (utilisation des couleurs blanche et bleue, hymnes produits en état de transe) assez proches. Ainsi, le panthéisme nordestin est lié à tout un ensemble de spiritualités et de croyances aujourd'hui très vivantes dans la société brésilienne ; quant à ses adeptes, ils ont contribué, comme ceux des cultes afro-brésiliens, tant traditionnels que syncrétiques, à la formation du Brésil moderne que nous connaissons.

\author{
Marion AUBRÉE \\ Centre de Recherches sur le Brésil Contemporain \\ EHESS
}

\title{
BIBLIOGRAPHIE
}

AUBRÉE Marion, Voyages entre corps et esprits - Étude comparative de deux courants religieux dans le Nordeste du Brésil, thèse de doctorat, Paris, Université Paris-VII, 1985.

Aubrée Marion, Laplantine François, La Table, le Livre et les Esprits - Naissance, évolution et actualité du mouvement social spirite entre France et Brésil, Paris, Lattès, 1990.

AubréE Marion, «Transe : entre libération de l'inconscient et contraintes socio-culturelles » in Maurice Godelier et J. Hassoun, Meurtre du Père - Sacrifice de la sexualité (Approches psychanalytiques et anthropologiques), Paris, Éditions Arcanes, 1996, pp. 173-192.

AUBRÉE Marion, «A constelação 'imaginal' ligada ao Conselheiro e outros carismáticos », communication présentée au Colloque «Canudos : cem anos de destruição », Salvador 30 sept./3 oct. 1997.

BASTIDE Roger, Le prochain et le lointain, Paris, Éditions Cujas, 1970.

Brown Diana, Umbanda, Religion and Politics in Urban Brazil, Michigan, UMI Research Press, 1986.

Cavalcanti Pedro, Arquivos de Assistencia a psicopatas de Pernambuco, Ano III, no 1, avril 1933, pp. 58-63.

Della Cava Ralph, Miracle at Joaseiro, Irvington (N.Y.), Columbia University Press, 1970.

FerRer-Benimeli (Père), Les archives secrètes du Vatican et la franc-maçonnerie, Paris, Dervy-Livres, 1989.

Ferretti Mundicarmo, Terra de Caboclo, São Luis de Maranhão, Planos Editorial, 1994.

Freyre Gilberto, Maîtres et esclaves, Paris, Gallimard, 1952 et 1974.

Gonçalves Fernandes F., Xangôs do Nordeste, investigações sobre os cultos negro-fetichistas do Recife, Rio de Janeiro, ed. Civilização Brasileira, 1937.

${ }^{34}$ Pour plus de détails sur ce mouvement religieux voir l'ouvrage d'Edward MACRAE, 1992. Cf. du même auteur, 1998, no 35-36, pp. 247-254. 
Gould Robert, Histoire abrégée de la franc-maçonnerie, Bruxelles, Éditions Lebègue, s.d.

Gouvea Mendonça Antonio, O celeste porvir, a inserção do protestantismo no Brasil, São Paulo, ed. Paulinas, 1984.

GueIRos VieIRa David, « Liberalismo, masonería y protestantismo en Brasil, siglo XIX » in Jean-Pierre Bastian, ed., Protestantes, liberales y francmasones, Mexico, CEHILA, 1990, p. 47.

Laplantine François, Nouss Alexis, Le métissage, Paris, Flammarion, 1997.

LÉvi-Strauss Claude, La pensée sauvage, Paris, Plon, 1962.

Lima Vicente, Xangô, Recife, 1937.

Macrae Edward, Guiado pela lua - Shamanismo e uso ritual da Ayahuasca no culto do Santo Daime, São Paulo, ed. Brasiliense, 1992.

MACRAE Edward, «L'utilisation religieuse de l'ayahuasca dans le Brésil contemporain », Cahiers du Brésil Contemporain, $\mathrm{n}^{\mathrm{0}} 35-36,1998$, pp. 247-254.

MARY André, Le défi du syncrétisme - le travail symbolique de la religion d'Eboga (Gabon), Paris, Édition de l'EHESS, 1999.

МоттA Roberto, «Indo-Afro-European syncretic cults in Brazil: their econnomic and social roots », Cahiers du Brésil Contemporain, no 5, déc. 1988, pp. 27-48.

Nina Rodrigues Raymundo, L'animisme fétichiste des nègres de Bahia (édité en français), Salvador de Bahia, Éd. Reis Campos, 1900.

Nina Rodrigues Raymundo, Os africanos no Brasil, œuvre posthume reconstituée à partir d'articles par Homero Pires, São Paulo, ed. Nacional, 1935, 5e édition, 1977.

Oliveira Viana F.J., Evolução do povo brasileiro, São Paulo, ed. Nacional, 1933.

Ortiz Renato, A morte branca do feiticeiro negro, Petropolis, éd. Vozes, 1978 ; thèse de doctorat présentée en français en 1975 : La mort blanche du sorcier noir, Paris, Éditions de l'EHESS.

Pereira De QueIroz Maria-Isaura, Réforme et révolution dans les sociétés traditionnelles, Paris, Anthropos, 1968 (éd. brésilienne, 1965).

Ramos Arthur, O negro brasileiro, etnografia religiosa, Rio de Janeiro, ed. Brasiliana, 1934.

RibeIRo René, Cultos afro-brasileiros do Recife, Recife, éd. IJNPS, 2è édition, 1978.

SANChIS Pierre, « Pra não dizer que não falei de sincretismo », Comunicações do ISER, ${ }^{\circ} 45,13 \mathrm{e}$ année, 1994, pp. 4-11.

Teixeira Monteiro Douglas, Os errantes do novo século, São Paulo, ed. Duas Cidades, 1974.

Torres Alberto, A organização nacional, São Paulo, ed. Nacional, 1933.

VAlEnsi Lucette, Fables de la mémoire - La glorieuse bataille des trois rois, Paris, Seuil, 1992.

VAlente Waldemar, « Panteismo em Pernambuco », Anuario da Faculdade de Filosofia 1962/64, vol. 7, Recife, 1966, pp. 129-179. 


\section{Résumé}

Dans le cadre d'une recherche plus large qui vise à comprendre s'il existe une spécificité de l'homo religiosus brésilien, cet article présente un mouvement, auto-désigné comme panthéiste, qui s'est développé de façon originale pendant quarante ans (années 1920-1960) au sein des populations relativement pauvres de cette ville. Après une présentation de la dynamique socioreligieuse ayant permis le surgissement d'un tel mouvement et l'exploration des divers courants qui ont pu contribuer à la formulation de la nouvelle doctrine, l'auteur analyse les documents qui permettent de reconstituer la doctrine et les pratiques des adeptes du Cercle. Après cela, l'auteur interroge les notions de bricolage, syncrétisme et métissage pour essayer de déterminer si elles sont susceptibles de rendre compte de cette création nouvelle qui répondait à la recherche de signes diacritiques capables de fonder une identité autre en même temps que de légitimer socialement des personnes qui tendaient à l'intégration dans la dynamique de construction nationale de l'époque.

\section{Abstract}

As part of a broader research aiming to identify a possible specificity of the Brasilian homo religiosus, the article focuses on a self-designated pantheistic movement which, for 40 years, experienced an original development among the disadvantaged population of Recife. After analysing the social and religious dynamics which led to the emergence of this movement, and the various trends which contributed to the formulation of the new doctrine, the author studies the documents which make it possible to reconstruct the members of the Circle's doctrine and practices. The author then questions the notions of " bricolage ", syncretism and "métissage " in order to determine whether they are likely to account for such a new creation which participated to the search for the diacritical signs that would form the basis of a new identity while, at the same time, offering a social legitimacy to people who aspired to being integrated into the dynamics of national construction then under way.

\section{Resumen}

En el marco de una investigación más amplia que apunta a comprender si existe una especificidad del homo religiosus brasilero, este artículo presenta un movimiento, auto-referito como panteísta, que se ha desarrollado de manera original, durante cuarenta años (años 1920-1960), en el seno de las poblaciones relativamente pobres de la ciudad de Recife. Luego de una presentación de la dinámica socio-religiosa que ha permitido el surgimiento del movimiento, y la exploración de las distintas corrientes que pudieron contribuir a la formulación de la nueva doctrina, la autora analiza los documentos que permiten reconstituir la doctrina y las prácticas de los adeptos del Círculo. A seguir son cuestionadas las nociones de bricolage, sincretismo y mestizaje para tratar de determinar si pueden dar cuenta de esta creación nueva que ha respondido a la búsqueda de signos diacríticos capaces de fundar una identidad otra, al mismo tiempo que legitima socialmente a personas que tenderían a la integración en la dinámica de construcción nacional de la época. 